\title{
MAXimizATion OF UTILITy AND PORTFOLIO SELECTION MODELS *
}

\author{
$\begin{array}{lll}\text { J. F. NEVES } & \text { P. N. DA SILVA } & \text { C. F. VASCONCELLOS } \\ & \end{array}$
}

\begin{abstract}
Modern portfolio theory deals with the combination of assets into a portfolio. It has diversification and maximization of expected utility as foundational principles. Its purpose is to find the portfolio which best meet the objectives of the investor. Markowitz [5] and Athayde e Flôres [2] have characterized the portfolios as solutions of constrained optimization problems. However, the relationship between the proposed problems and the utility maximization principle is not clear. Taking into account the results of Scott and Horvath [10], we prove that such problems correspond to the maximization of the expected utility of the investor that underlies each model.
\end{abstract}

\section{Introduction}

A portfolio consists of several assets selected for investment gains. The portfolio selection may be divided into two stages: the analysis of available assets and the combination of selected assets into a portfolio. The modern portfolio theory deals with the second stage.

Utility theory is the foundation for the theory of choice under uncertainty. A utility function measures investor's relative preference for different levels of total wealth. For von Neumann and Morgenstern [7], a rational investor selects, among a set of competing feasible investment alternatives, an investment which maximizes his expected utility of wealth (see Rose [8]).

In Markowitz [5], the optimal portfolio minimizes the risk for a given level of return. In Athayde and Flôres [2], the optimal portfolio minimizes the risk for given levels of return and skewness. None of these models addresses investor's expected utility. The relationship between the proposed optimization problems and the utility maximization principle is not clear. Taking into account the results of Scott and Horvath [10], we analyze the maximization of the expected utility underlying the models of Markowitz [5] and Athayde and Flôres [2].

The paper is organized as follows: Section 2 deals with the Markowitz [5] and Athayde and Flôres [2] approaches. In Section 3, we briefly present Scott and Horvath [10] results on preferences on distribution moments. Finally, in Section 4, we analyze the underlying expected utility of the models of Markowitz [5] and Athayde and Flôres [2].

\footnotetext{
${ }^{*}$ The first author is supported by CAPES

Key words: Expected utility. Portfolio selection. Odd and even moments.

†discente no Programa de Pós-graduação em Ciências Computacionais, IME/UERJ, nevesfra@gmail.com

‡docente no Departamento de Análise Matemática, IME/UERJ, nunes@ime.uerj.br; This author is partially supported by FAPERJ grants E26/010.002646/2014 and E-26/203.537/2015

§pesquisador visitante, IME/UERJ/FAPERJ, cfred@ime.uerj.br; This author is partially supported by FAPERJ grants E26/010.002646/2014 and E-26/203.537/2015
}

DOI: 10.12957/cadmat.2017.29731 


\section{Portfolio Selection Models}

In this section we describe Markowitz [5] and Athayde and Flôres [2] portfolio selection models. In both models, short sales are allowed and they consider $n$ risky assets and a riskless one. The return $R_{i}$ of the $i$-th asset is a random variable with mean $\mu_{i}$ and $r_{f}$ is the risk free rate of return. Let $\mathbf{R}$ denote the $n \times 1$ vector whose $i$-th element is $R_{i}$ and $\mathbf{M}_{1}$ denote the $n \times 1$ vector whose $i$-th element is $\mu_{i}$. Let $\mathbf{M}_{2}$ denote the symmetric $n \times n$ matrix whose $(i, j)$-th element is the covariance $\sigma_{i j}$ between the two random variables $R_{i}$ and $R_{j}$. Notice that $\mathbf{M}_{1}$ and $\mathbf{M}_{2}$ stand for the matrices containing the expected returns and covariances of the random vector $\mathbf{R}$ of $n$ risky assets. That is $\mathbf{M}_{1}$ is the expected value of $\mathbf{R}$ and $\mathbf{M}_{2}$, its variance $\operatorname{Var}(\mathbf{R})=E\left[\left(\mathbf{R}-\mathbf{M}_{1}\right)\left(\mathbf{R}-\mathbf{M}_{1}\right)^{t}\right]$, where ${ }^{t}$ is the symbol for transposition and $E[\mathbf{X}]$ is the expected value of the random variable $\mathbf{X}$. Let $\mathbf{M}_{3}$ denote the $n \times n^{2}$ matrix whose elements are the skewnesses of the random vector $\mathbf{R}$. That is a generic element $\sigma_{i j k}$ of $\mathbf{M}_{3}$ is given by $\sigma_{i j k}=E\left[\left(R_{i}-\mu_{i}\right)\left(R_{j}-\mu_{j}\right)\left(R_{k}-\mu_{k}\right)\right]$ and $\mathbf{M}_{3}=E\left[\left(\mathbf{R}-\mathbf{M}_{1}\right)\left(\mathbf{R}-\mathbf{M}_{1}\right)^{t} \otimes\left(\mathbf{R}-\mathbf{M}_{1}\right)^{t}\right]$, where $\otimes$ denotes the Kronecker (tensor) product. If [1] stands for the $n \times 1$ vector of 1 's, the expected (excess) return $\mathbf{x}$ of the random vector of $n$ assets $\mathbf{R}$ is given by $\mathbf{x}=\mathbf{M}_{1}-[1] r_{f}$.

The assets are combined in the portfolio in some proportion. Let $\alpha \in \mathbb{R}^{n}$ a vector of weights. Notice that each component $\alpha_{i}$ of $\alpha$ is the number of units (shares) held of asset $i$. The mean return, variance and skewness of the portfolio with these weights will be, respectively: $\alpha^{t} \mathbf{x}, \alpha^{t} \mathbf{M}_{2} \alpha$ and $\alpha^{t} \mathbf{M}_{3}(\alpha \otimes \alpha)$.

Markowitz [5] considers the two first moments (mean and variance) in his portfolio selection model. If short sales are allowed, the investor portfolio corresponds to finding vector of weights $\alpha$ on the risky assets that minimizes the variance for a given expected return $E\left(r_{p}\right)$. Calling $R$ the given (excess) portfolio return $E\left(r_{p}\right)-r_{f}$, the mean-variance efficient portfolio is the solution of the constrained optimization problem:

$$
\left\{\begin{array}{l}
\min _{\alpha} \alpha^{t} \mathbf{M}_{2} \alpha \\
\alpha^{t} \mathbf{x}=R
\end{array}\right.
$$

Athayde and Flôres [2] consider the three first moments (mean, variance and skewness) and allow short sales. They characterize the efficient portfolios set for $n$ risky assets and a riskless one under the assumption that agents like odd moments and dislike even ones. The investor portfolio corresponds to finding vector of weights $\alpha$ on the risky assets that minimizes the variance for a given expected return $E\left(r_{p}\right)$ and skewness $\sigma_{p^{3}}$. As before, calling $R$ the given (excess) portfolio return $E\left(r_{p}\right)-r_{f}$, the mean-variance-skewness efficient portfolio is a solution of the constrained optimization problem:

$$
\left\{\begin{array}{l}
\min _{\alpha} \alpha^{t} \mathbf{M}_{2} \alpha \\
\alpha^{t} \mathbf{x}=R \\
\alpha^{t} \mathbf{M}_{3}(\alpha \otimes \alpha)=\sigma_{p^{3}}
\end{array}\right.
$$

Since mean-variance analysis takes into account only the first two moments, it is consistent with expected utility maximization if either investors have quadratic utility or portfolio returns are normally 
distributed, i.e. the moments with order strictly greater than two are null. (Amenc and Le Sourd [1]). Similarly, in Athayde and Flôres model, we have an utility fucntion fully described by the three first moments: mean, variance and skewness. In [5], Markowitz presents the solution of the convex quadratic problem (2.1). For results on the existence of solution to (2.2), see Martins, Vasconcellos and Silva [6]

\section{$3 \quad$ Directions of preference for moments}

In this section, we briefly present results on preferences on distribution moments developed by Scott and Horvath [10]. Let $w_{0}$ be the initial investor's wealth and $r$ a random variable representing relative return on investiment. The investor's utility function $U=U\left(w_{0}+r w_{0}\right)$ quantifies the utility to an investor of the relative return $r$ on initial wealth $w_{0}$. Let $\mu$ denote the mean of $w_{0}+w_{0} r$. They expand the utility function $U$ in a Taylor series around $\mu$ and take the expected value to obtain

$$
E(U)=U(\mu)+\sum_{i=2}^{\infty} \frac{\mu_{i}}{i !} U^{(i)}(\mu) .
$$

where $U^{(i)}$ denotes the $i$-th derivative of $U$ and $\mu_{i}$ is the $i$-th central moment.

Theorem 3.1 (Theorem 1, Scott and Horvath [10]). Investors exhibiting positive marginal utility of wealth for all wealth levels, consistent risk aversion at all wealth levels, and strict consistency of moment preference will have positive preference for positive skewness (negative preference for negative skewness).

Strict consistency of moment preference means that the coefficient of the $i$-th moment in (3.3) will always be positive, zero, or negative regardless of wealth level. The assumptions of Theorem 3.1 can be expressed by means of the investor's utility function as

- $U^{\prime}(w)>0$, for all $w$ (positive marginal utility)

- $U^{\prime \prime}(w)<0$, for all $w$ (consistent risk aversion)

and characterize the usual risk averse investor.

Also, having positive preference for positive skewness (negative preference for negative skewness) means that $U^{\prime \prime \prime}(w)>0$, for all $w$.

For a discussion on the convergence of the infinite Taylor series expansion to the expected utility, see Lhabitant [3] and Loistl [4].

\section{Maximization of expected utility}

Using an axiomatic approach, von Neumann and Morgenstern [7] proved that rational choices in uncertain situations can be represented by a utility function. Utility functions are unique up to positive affine transformation (multiplication by a positive number and addition of any scalar) (see Rubinstein [9]). The

DOI: $10.12957 /$ cadmat.2017.29731 
relationship between Markowitz [5] and Athayde and Flôres [2] optimization problems and the utility maximization principle is not clear. In Theorems 4.1 and 4.2, we overcome this lack of connection.

In Markowitz model, the moments with order strictly greater than two are null. Thus using the uniqueness up to positive affine transformation, we may assume from (3.3) that its underlying expected utility function $E_{M}(U)$ is given by

$$
E_{M}(U)=U\left(\mu_{1}\right)+\frac{U^{\prime \prime}\left(\mu_{1}\right)}{2} \mu_{2} .
$$

Similarly, in Athayde and Flôres [2] model, we may assume from (3.3) that the underlying expected utility function $E_{F}(U)$ is given by

$$
E_{F}(U)=U\left(\mu_{1}\right)+\frac{U^{\prime \prime}\left(\mu_{1}\right)}{2} \mu_{2}+\frac{U^{\prime \prime \prime}\left(\mu_{1}\right)}{6} \mu_{3} .
$$

To study the behavior of the expected utility functions, we consider them as functions of the central moments $\mu_{i}(\mathrm{i}=1,2, \ldots)$. Throughout this section, we assume positive marginal utility, decreasing absolute risk aversion at all wealth levels together with strict consistency for moment preference.

Theorem 4.1. Let the expected utility function $E_{M}(U)$ as in (4.4). To maximize $E_{M}(U)$ with a given expected return ( $\mu_{1}$ is fixed), it is necessary and sufficient to minimize the variance $\mu_{2}$.

Proof. Since $\mu_{1}$ is fixed and $U^{\prime \prime}\left(\mu_{1}\right)<0$ (decreasing absolute risk aversion), to maximize $E_{M}(U)$ in (4.4) it is necessary and sufficient to minimize the variance $\mu_{2}$.

Theorem 4.2. Let the expected utility function $E_{F}(U)$ as in (4.5). Then,

1. to maximize $E_{F}(U)$ with a given expected return and skewness ( $\mu_{1}$ and $\mu_{3}$ are fixed), it is necessary and sufficient to minimize the variance $\mu_{2}$.

2. to maximize $E_{F}(U)$ with a given expected return and variance ( $\mu_{1}$ and $\mu_{2}$ are fixed), it is necessary and sufficient to maximize the skewness $\mu_{3}$.

Proof. 1. Since $\mu_{1}$ and $\mu_{3}$ are fixed, to maximize $E_{F}(U)$ in (4.5) it is necessary and sufficient to maximize $\frac{U^{\prime \prime}\left(\mu_{1}\right)}{2} \mu_{2}$. Decreasing absolute risk aversion assumption implies that $U^{\prime \prime}\left(\mu_{1}\right)<0$. Thus the maximum is achieved if and only if we minimize the variance $\mu_{2}$.

2. Since $\mu_{1}$ and $\mu_{2}$ are fixed, to maximize $E_{F}(U)$ in (4.5) it is necessary and sufficient to maximize $\frac{U^{\prime \prime \prime}\left(\mu_{1}\right)}{6} \mu_{3}$. From Theorem 3.1, we have $U^{\prime \prime \prime}\left(\mu_{1}\right)>0$. Thus the maximum is achieved if and only if we maximize the skewness.

\section{$5 \quad$ Final Remarks}

We have used the results of [10] to establish a connection between the constrained optimization problems proposed by Markowitz [5] and Athayde e Flôres [2] and the maximization of expected utility principle. 
We have proved that such optimization problems correspond to the maximization of the expected utility of the investor underlying each of the models. 


\section{References}

[1] AMEnC, N.; LE SOURD, V. Portfolio Theory and Performance Analysis. John Wiley \& Sons, England, 2005.

[2] ATHAYDE, G. M. de; FLÔRES JR., R. G. Finding a maximum skewness portfolio - a general solution to three moments portfolio choice. Journal of Economic Dynamics and Control, v. 28, 2004, p. $1335-1352$.

[3] LHABITANT, F. S. On the (ab)use of Taylor series approximations for portfolio selection, portfolio performance and risk management. Working Paper, University of Lausanne, 1998, p. 1-23.

[4] LOISTL, O. The erroneous approximation of expected utility by means of a Taylor's series expansion: analytic and computational results. The American Economic Review, v. 66, n. 5, 1976, p. 904-910.

[5] MARKOWITZ, H. Portfolio Selection. The Journal of Finance, v. 7, 1952, p. 77-91.

[6] MARTINS, P. R.; VASCONCELlOS, C. F.; SILVA, P. N. Análise de Modelos de Seleção de Carteiras de Investimento. Cadernos do IME - Série Matemática, v. 8, 2014, p. 11-38.

[7] NEUMAnN, J. von; MORGEnSTERN, O. Theory of games and economic behavior. Princeton University Press, New Jersey, 1944.

[8] ROSE, M. Reward Management. Kogan Page, London, 2014.

[9] RUBinstein, A. Lecture Notes in Microeconomic Theory. The Economic Agent. Princeton University Press, New Jersey, 2006.

[10] SCOTT, R. C.; HORVATH, P. A. On the direction of preference for moments of higher order than the variance. The Journal of Finance, v. 35, n. 4, 1980, p. 915-919. 